\title{
Interaction among systems of finite size in predictive relativistic mechanics. II. Electromagnetic and gravitational interactions
}

\author{
Xavier Fustero and Enric Verdaguer \\ Departament de Física Teòrica and Grupo Interuniversitario de Física Teòrica, Universitat Atòmona de Barcelona, Bellaterra \\ (Barcelona), Spain \\ (Received 10 October 1979; revised manuscript received 3 August 1981)
}

\begin{abstract}
We explicitly construct a closed system of differential equations describing the electromagnetic and gravitational interactions among bodies to first order in the coupling constants, retaining terms up to order $c^{-2}$. The Breit and Barker and O'Connell Hamiltonians are recovered by means of a coordinate transformation. The method used throws light on the meaning of these coordinates.
\end{abstract}

\section{INTRODUCTION}

As far as we know predictive relativistic mechanics (PRM) has been able to give a satisfactory account of a great variety of interactions among structureless particles within the framework of perturbation theory. It is therefore tempting to try to build a framework able to describe the interactions among particles possessing an internal structure taking as a background PRM. In the preceding paper, ${ }^{1}$ hereafter referred to as I, we have given a systematic way of constructing a set of dynamical equations, up to first order in the coupling constants for objects possessing an internal structure, the energy, center-of-mass position, and spin of each system, defined as if each were an isolated system. The time evolution of these quantities due to the interactions was then calculated using PRM. The system of differential equations thus obtained becomes closed under the assumption of finite multipolar structure for the interacting objects if we provide the equations of motion for the multipolar moments used.

The next logical step is to test these dynamical equations by studying specific interactions. On one hand, this will give a test for the definitions of center of mass and spin given in I and, on the other hand, it could give information about the possibility of building a classical, as opposed to quantum, model for spinning particles. This is the reason why the electromagnetic and gravitational interactions have been chosen first. The quantized version of the electromagnetic theory is the best tested of the known interactions and in the domain of gravitational interactions the postNewtonian effects for spinning bodies will probably be measured in the near future by satellite experiments or from the observation of astrophysical objects such as the pulsar PSR $1913+16$.

We have limited our study to the simpler case. We have assumed rigid-body behavior and nearly spherical distribution for each subsystem. The calculations have only been carried out up to order $c^{-2}$. For the electromagnetic interaction this has resulted, of course, in a great simplification in the calculations, but on the other hand it has allowed a direct comparison with the Breit Hamiltonian. The case of the gravitational interaction is a different one. Within the framework of PRM the only candidate for this interaction is the Einstein-Infeld-Hoffmann Lagrangian, which contains only terms up to $c^{-2}$.

As the multipolar moments are defined in the rest system of each body, in order to get covariant expressions for them, the transformation relating the relative coordinates in the observer frame and the rest frame must be studied. This is done in Sec. II where the basic assumptions on the finite multipolar structure we use are also introduced

In order to avoid unnecessary calculations it is useful to study the structure of the dynamical equations when expanded up to terms of order $c^{-2}$. This is done in Sec. III.

These are all the tools needed in order to find the equations for the electromagnetic interaction, which are derived in Sec. IV. To this order the mass can be taken as constant and the equation giving the time derivative of the spin agrees with the equation of Bargmann, Michel, and Telegdi. The system is closed assuming $\vec{\mu}_{i}=g_{i}\left(Q_{i} /\right.$ $\left.M_{i}\right) \vec{S}_{i}$ and the equations giving the acceleration of the center of mass contains terms derived from the Darwin Lagrangian plus spin-orbit and spinspin coupling terms.

The equations for the gravitational interaction are obtained in Sec. V. The mass can also be taken as constant and our equation for the spin contains the same terms as the equation of Cho and Hari Dass ${ }^{2}$ and of Barker and O'Connell, ${ }^{3}$ plus an extra term that introduces a change in the magnitude of the spin. The equation for the acceleration of the center of mass coincides with the one given by Pirani ${ }^{4}$ for a gyroscope orbiting a mas- 
sive body if we take the limit $\vec{V}_{2}=0$.

Section VI is devoted to a further study of the equations giving the acceleration of the center of mass. We investigate the possibility of deriving them from a Lagrangian. The result we find is that these equations cannot be derived from a Lagrangian, but it can be seen that if instead of the center of mass we use the center of spin, then its acceleration is given by an equation derivable from a Lagrangian in both cases. If one performs a Legendre transformation on these Lagrangians one finds the classical version of the Breit Hamiltonian in the case of the electromagnetic interaction and the Hamiltonian of Barker and O'Connell ${ }^{5}$ for the gravitational interaction.

The meaning of the different sets of equations and the higher-order corrections are discussed in the conclusions, in Sec. VII.

\section{MULTIPOLAR EXPANSION}

In the equations of motion for bodies of finite size, given in I, a multipolar expansion must be introduced in the functions $\dot{\Lambda}$ and $\ddot{\Lambda}$. This multipolar expansion is performed around the center of mass of the bodies, $\overrightarrow{\mathrm{X}}_{1}$ and $\overrightarrow{\mathrm{X}}_{2}$ and the multipolar moments are defined in the rest system of each body.

Let us now introduce the notations that will be used in the sequel. Given a system of particles whose positions and velocities at $t=0$ in a given system of reference are $x_{a}^{i}$ and $v_{a}^{i}$, respectively, the positions and velocities of these particles at the instant $t^{\prime}=0$ in another system of reference moving with velocity $V_{1}$ with respect to the former are given as functions of $x_{a}^{i}$ and $v_{a}^{i}$ by the relations

$\overrightarrow{\mathbf{x}}_{a}^{\prime}=\overrightarrow{\mathrm{x}}_{a}+\frac{\overrightarrow{\mathrm{x}}_{a} \cdot \overrightarrow{\mathrm{V}}_{1}}{1-\overrightarrow{\mathrm{v}}_{a} \cdot \overrightarrow{\mathrm{V}}_{1}}\left(\overrightarrow{\mathrm{v}}_{a}+\frac{\gamma^{-1}-1}{V_{1}^{2}} \overrightarrow{\mathrm{V}}_{1}\right), \quad \gamma=\left(1-V_{1}^{2}\right)^{-1 / 2}$,

$\overrightarrow{\mathrm{v}}_{a}^{\prime}=\frac{\overrightarrow{\mathrm{V}}_{a}+\overrightarrow{\mathrm{V}}_{1}\left[\frac{\overrightarrow{\mathrm{V}}_{1} \cdot \overrightarrow{\mathrm{V}}_{a}}{V_{1}^{2}}(\gamma-1)-\gamma\right]}{\left(1-\overrightarrow{\mathrm{V}}_{1} \cdot \overrightarrow{\mathrm{v}}_{a}\right)}$,

in the case of a system of free particles. In this case the center of mass in its rest frame is given by

$$
\overrightarrow{\mathrm{X}}_{1}^{\prime}=\overrightarrow{\mathrm{X}}_{1}+\frac{\overrightarrow{\mathrm{X}}_{1} \cdot \overrightarrow{\mathrm{V}}_{1}}{V_{1}^{2}}(\gamma-1) \overrightarrow{\mathrm{V}}_{1}
$$

From these expressions the relation between $\vec{r}_{a}$ $\equiv \overrightarrow{\mathrm{X}}_{a}^{\prime}-\overrightarrow{\mathrm{X}}_{1}^{\prime}$ and $\overrightarrow{\mathbf{r}}_{a} \equiv \overrightarrow{\mathrm{X}}_{a}-\overrightarrow{\mathrm{X}}_{1}$ can easily be calculated.

In the general case of interacting particles, in order to compute the analog of (2.1), (2.2), and (2.3) the knowledge of the world line of the particles is needed. In our case, however, as the relation between $\overrightarrow{\mathbf{r}}_{a}$ and $\overrightarrow{\mathbf{x}}_{a}$ is needed only to write the functions $\dot{\Lambda}$ and $\ddot{\Lambda}$ as functions of the multi- polar moments, and as these functions are of first order in the coupling constants, only the zeroth-order terms of these relations are needed, which are given by (2.1)-(2.3).

Since in the remaining part of this paper the calculations will only be performed up to order $c^{-2}$, we shall give these relations only up to this order,

$$
\begin{aligned}
& \overrightarrow{\mathrm{x}}_{a}^{\prime}=\overrightarrow{\mathrm{x}}_{a}+\frac{1}{c^{2}}\left(\overrightarrow{\mathrm{x}}_{a} \cdot \overrightarrow{\mathrm{V}}_{1}\right)\left(\overrightarrow{\mathrm{V}}_{a}-\frac{1}{2} \overrightarrow{\mathrm{V}}_{1}\right) \\
& \overrightarrow{\mathrm{X}}_{1}^{\prime}=\overrightarrow{\mathrm{X}}_{1}+\frac{1}{2 c^{2}}\left(\overrightarrow{\mathrm{X}}_{1} \cdot \overrightarrow{\mathrm{V}}_{1}\right) \overrightarrow{\mathrm{V}}_{1} .
\end{aligned}
$$

Therefore we have

$$
\begin{aligned}
\overrightarrow{\mathbf{x}}_{a}= & \overrightarrow{\mathrm{r}}_{a}-\frac{1}{2 c^{2}}\left(\overrightarrow{\mathrm{r}}_{a} \cdot \overrightarrow{\mathrm{V}}_{1}\right) \overrightarrow{\mathrm{V}}_{1}-\frac{1}{c^{2}}\left(\overrightarrow{\mathrm{r}}_{a} \cdot \overrightarrow{\mathrm{V}}_{1}\right) \overrightarrow{\mathrm{W}}_{a} \\
& -\frac{1}{c^{2}}\left(\overrightarrow{\mathrm{X}}_{1} \cdot \overrightarrow{\mathrm{V}}_{1}\right) \overrightarrow{\mathrm{w}}_{a}
\end{aligned}
$$

and

$$
\begin{aligned}
\overrightarrow{\mathbf{r}}_{A}= & \overrightarrow{\mathrm{r}}_{A}-\frac{1}{2 c^{2}}\left(\overrightarrow{\mathrm{r}}_{A} \cdot \overrightarrow{\mathrm{V}}_{2}\right) \overrightarrow{\mathrm{V}}_{2}-\frac{1}{c^{2}}\left(\overrightarrow{\mathrm{r}}_{A} \cdot \overrightarrow{\mathrm{V}}_{2}\right) \overrightarrow{\mathrm{w}}_{A} \\
& -\frac{1}{c^{2}}\left(\overrightarrow{\mathrm{X}}_{2} \cdot \overrightarrow{\mathrm{V}}_{2}\right) \overrightarrow{\mathrm{w}}_{A}
\end{aligned}
$$

for the relative positions of systems 1 and $2, \overrightarrow{\mathrm{w}}_{a}$ and $\overrightarrow{\mathrm{w}}_{A}$ being $\overrightarrow{\mathrm{v}}_{a}-\overrightarrow{\mathrm{V}}_{1}$ and $\overrightarrow{\mathrm{v}}_{A}-\overrightarrow{\mathrm{V}}_{2}$, respectively.

One of the basic assumptions used in this work is that each subsystem behaves as a rigid body, therefore a slight correction of (2.6) and (2.7) must be introduced. This can easily be seen by studying the meaning of the term $c^{-2}\left(\overrightarrow{\mathrm{X}}_{1} \cdot \overrightarrow{\mathrm{V}}_{1}\right) \overrightarrow{\mathrm{w}}_{a}$. This term is the correction due to the action of the relative velocity $\overrightarrow{\mathrm{w}}_{a}$ along the time $c^{-2}\left(\overrightarrow{\mathrm{X}}_{1} \cdot \overrightarrow{\mathrm{V}}_{1}\right)$; as this time is long in general, the mean value of $\overrightarrow{\mathrm{w}}_{a}$ must be used. For a solid body this mean value is zero, therefore, in our case the last term on the right-hand sides of Eqs. (2.6) and (2.7) vanishes and the relations we must use are

$$
\begin{aligned}
& \overrightarrow{\mathrm{r}}_{a}=\overrightarrow{\mathrm{r}}_{a}-\frac{1}{2 c^{2}}\left(\overrightarrow{\mathrm{r}}_{a} \cdot \overrightarrow{\mathrm{V}}_{1}\right) \overrightarrow{\mathrm{V}}_{1}-\frac{1}{c^{2}}\left(\overrightarrow{\mathrm{r}}_{a} \cdot \overrightarrow{\mathrm{V}}_{1}\right) \overrightarrow{\mathrm{w}}_{a}, \\
& \overrightarrow{\mathrm{r}}_{A}=\overrightarrow{\mathrm{r}}_{A}-\frac{1}{2 c^{2}}\left(\overrightarrow{\mathrm{r}}_{A} \cdot \overrightarrow{\mathrm{V}}_{2}\right) \overrightarrow{\mathrm{V}}_{2}-\frac{1}{c^{2}}\left(\overrightarrow{\mathrm{r}}_{A} \cdot \overrightarrow{\mathrm{V}}_{2}\right) \overrightarrow{\mathrm{w}}_{A} .
\end{aligned}
$$

The rigid-body assumption coupled with spherical symmetry gives also

$$
\vec{\delta}_{1}=\epsilon^{a} \overrightarrow{\mathrm{r}}_{a}=0, \quad \frac{d}{d t} \vec{\delta}_{1}=0
$$

and

$$
D^{i j}=D \delta^{i j} \Rightarrow \frac{d D^{i j}}{d t}=\epsilon^{a} e_{a}\left(r_{a}^{i} w_{a}^{j}+r_{a}^{j} w_{a}^{i}\right)=0
$$

This relation implies the antisymmetry of

$$
M_{1}^{i j}=\epsilon_{a} e_{a} r_{a}^{i} w_{a}^{j}=\frac{1}{2} \epsilon_{a}\left(r_{a}^{i} w_{a}^{j}-r_{a}^{j} w_{a}^{i}\right) .
$$


Therefore, defining

$$
\vec{\mu}_{1}=\epsilon^{a} e_{a} \overrightarrow{\mathrm{r}}_{a} \times \overrightarrow{\mathrm{w}}_{a},
$$

$M_{1}^{i j}$ can be written as

$$
M_{1}^{i j}=\frac{1}{2} \delta^{i j k} \mu_{i k} .
$$

If small departures from spherical symmetry are allowed we can use

$$
D^{i j}=D \delta^{i j}+\epsilon \gamma^{i j},
$$

where $\epsilon$ is a small parameter. Then we shall have

$$
M_{1}^{i j}=\frac{1}{2} \delta^{i j k} \mu_{l k}+O(\hat{\epsilon}) .
$$

The terms of order $\epsilon c^{-2}$ will be neglected.

Static moments of order higher than the dipolar. and kinetic moments of order higher than $M^{i j}$ will be neglected.

III. EQUATIONS UP TO ORDER $c^{-2}$

Let us now write down the Eqs. (8.8), (8.9), and (8.10) of I retaining only terms up to order $c^{-2}$. We find for the mass

$$
\frac{d M_{i}}{d t}=c^{-2}\left(\dot{H}_{i}-\overrightarrow{\mathrm{V}}_{i} \cdot \dot{\overrightarrow{\mathrm{P}}}_{i}\right) \text {. }
$$

The equation for the spin becomes

$$
\frac{d \overrightarrow{\mathrm{S}}_{i}}{d t}=c^{-2}\left\{\dot{\mathrm{H}}_{i}\left(\overrightarrow{\mathrm{X}}_{i} \times \overrightarrow{\mathrm{V}}_{i}\right)-\left(\dot{\overrightarrow{\mathrm{J}}} i \cdot \overrightarrow{\mathrm{V}}_{i}\right) \overrightarrow{\mathrm{V}}_{i}+\frac{1}{M_{i}}\left[\left(\dot{\overrightarrow{\mathrm{P}}}_{i} \cdot \overrightarrow{\mathrm{S}}_{i}\right) \overrightarrow{\mathrm{V}}_{i}-\left(\overrightarrow{\mathrm{S}}_{i} \cdot \overrightarrow{\mathrm{V}}_{i}\right) \dot{\overrightarrow{\mathrm{P}}}_{i}\right]-\frac{1}{2}\left[\dot{\overrightarrow{\mathrm{P}}}_{i} \cdot\left(\overrightarrow{\mathrm{X}}_{i} \times \overrightarrow{\mathrm{V}}_{i}\right)\right] \overrightarrow{\mathrm{V}}_{i}\right\}+\left(1+\frac{V_{i}^{2}}{2 c^{2}}\right)\left(\dot{\overrightarrow{\mathrm{J}}}_{i}-\overrightarrow{\mathrm{X}}_{i} \times \dot{\overrightarrow{\mathrm{P}}}_{i}\right)
$$

And the equation for the center of mass becomes

$$
\begin{aligned}
\frac{d^{2} \overrightarrow{\mathrm{X}}_{i}}{d t^{2}}= & \left(1+\frac{V_{i}^{2}}{2 c^{2}}\right) M_{i}^{-1}\left(\dot{\overrightarrow{\mathrm{P}}}_{i}+\ddot{\overrightarrow{\mathrm{K}}}_{i}\right) \\
& +\frac{1}{c^{2} M_{i}}\left[\left(\ddot{\overrightarrow{\mathrm{K}}}_{i} \cdot \overrightarrow{\mathrm{V}}_{i}\right) \overrightarrow{\mathrm{V}}_{i}-\ddot{H}_{i} \overrightarrow{\mathrm{X}}_{i}-\frac{\ddot{\overrightarrow{\mathrm{P}}}_{i} \times \overrightarrow{\mathrm{S}}_{i}}{M_{i}}+\left(\ddot{\overrightarrow{\mathrm{P}}}_{i} \cdot \overrightarrow{\mathrm{V}}_{i}\right) \overrightarrow{\mathrm{V}}_{i}-\left(\overrightarrow{\mathrm{V}}_{i} \cdot \overrightarrow{\mathrm{X}}_{i}\right) \ddot{\overrightarrow{\mathrm{P}}}_{i}+\ddot{\overrightarrow{\mathrm{J}}}_{i} \times \ddot{\overrightarrow{\mathrm{V}}}_{i}-2 \dot{\mathrm{H}}_{i} \overrightarrow{\mathrm{V}}_{i}+\left(\dot{\overrightarrow{\mathrm{P}}}_{i} \cdot \overrightarrow{\mathrm{V}}_{i}\right) \overrightarrow{\mathrm{V}}_{i}-2 V_{i} \dot{\overrightarrow{\mathrm{P}}}_{i}\right] .
\end{aligned}
$$

Therefore in order fo find $(3.1)-(3.3)$ the expressions of $\overrightarrow{\mathrm{J}}_{i}, \overrightarrow{\overrightarrow{\mathrm{P}}}_{i}, \overrightarrow{\overrightarrow{\mathrm{K}}}_{i}$, and $\overrightarrow{\mathrm{K}}_{i}$ must be computed up to order $c^{-2}$, whereas $\dot{H}_{i}, \ddot{H}_{i}, \overrightarrow{\mathrm{P}}_{i}$, and $\overrightarrow{\mathrm{J}}_{i}$ must be computed only to order zero. We shall now write these expressions for specific interactions.

\section{ELECTROMAGNETIC INTERACTION}

The first step to find the dynamical equations for electromagnetically interacting bodies is the cal- culation of $H_{i}, \overrightarrow{\mathrm{P}}_{i}, \overrightarrow{\mathrm{J}}_{i}$, and $\overrightarrow{\mathrm{K}}_{i}$. This can be made, of course, using the integral equations given in $\mathrm{I}$ for these quantities to find them up to first order in the coupling constants, and then performing a $v / c$ expansion. However, in this case we can use an equivalent and even shorter procedure because we have the Darwin Lagrangian at our disposal.

In any case the result is

$$
\begin{aligned}
& H_{1}=\epsilon^{a} m_{a} c^{2}+\frac{1}{2} \epsilon^{a} m_{a} v_{a}^{2}+\frac{1}{2} \epsilon^{a} \epsilon^{a^{\prime}} \frac{e_{a} e_{a^{\prime}}}{x_{a a^{\prime}}} \\
& +c^{-2}\left\{\frac{3}{8} \epsilon^{a} m_{a} v_{a}^{4}+\frac{1}{4} \epsilon^{a} \epsilon^{a^{\prime}} \frac{e_{a} e_{a^{\prime}}}{x_{a a^{\prime}}}\left[\overrightarrow{\mathrm{v}}_{a} \cdot \overrightarrow{\mathrm{v}}_{a^{\prime}}+\frac{\left(\overrightarrow{\mathrm{x}}_{a a^{\prime}} \cdot \overrightarrow{\mathrm{v}}_{a}\right)\left(\overrightarrow{\mathrm{x}}_{a a^{\prime}} \cdot \overrightarrow{\mathrm{v}}_{a^{\prime}}\right)}{x_{a a^{\prime}}^{2}}\right]\right\}, \\
& \overrightarrow{\mathrm{P}}_{1}=\epsilon^{a} m_{a} \overrightarrow{\mathrm{v}}_{a}+\frac{1}{2 c^{2}}\left[\epsilon^{a} m_{a} v_{a}^{2} \overrightarrow{\mathrm{v}}_{a}+\epsilon^{a} \epsilon^{a^{\prime}} \frac{e_{a} e_{a^{\prime}}}{x_{a a^{\prime}}}\left(\overrightarrow{\mathrm{v}}_{a^{\prime}}+\frac{\overrightarrow{\mathrm{x}}_{a a^{\prime}} \cdot \overrightarrow{\mathrm{v}}_{a^{\prime}}}{x_{a a^{\prime}}^{2}} x_{a a^{\prime}}\right)\right] \text {, } \\
& \overrightarrow{\mathrm{J}}_{1}=\epsilon^{a} m_{a} \overrightarrow{\mathrm{x}}_{a} \times \overrightarrow{\mathrm{v}}_{a}+\frac{1}{2 c^{2}}\left[\epsilon^{a} \overrightarrow{\mathrm{x}}_{a} \times m_{a} v_{a}^{2} \overrightarrow{\mathrm{v}}_{a}+\epsilon^{a} \epsilon^{a^{\prime}} \frac{e_{a} e_{a^{\prime}}}{x_{a a^{\prime}}} \overrightarrow{\mathbf{x}}_{a} \times\left(\overrightarrow{\mathrm{v}}_{a^{\prime}}+\frac{\overrightarrow{\mathrm{x}}_{a a^{\prime}} \cdot \overrightarrow{\mathrm{v}}_{a^{\prime}}}{x_{a a^{\prime}} \overrightarrow{\mathrm{x}}_{a a^{\prime}}}\right)\right] \text {, } \\
& \overrightarrow{\mathrm{K}}_{1}=\epsilon^{a} m_{a} \overrightarrow{\mathrm{x}}_{a}+\frac{1}{2 c^{2}}\left(\epsilon^{a} m_{a} v_{a}^{2} \overrightarrow{\mathrm{x}}_{a}+\epsilon^{a} \epsilon^{a^{\prime}} e_{a} e_{a^{\prime}} \frac{\overrightarrow{\mathrm{x}}_{a}}{\overrightarrow{\mathrm{x}}_{a a^{\prime}}}\right) \text {. }
\end{aligned}
$$

We must find now $\overrightarrow{\mathrm{K}}_{1}, \dot{\overrightarrow{\mathrm{P}}}_{1}, \dot{\vec{J}}_{1}$, and $\ddot{\overrightarrow{\mathrm{K}}}_{1}$ up to terms of order $c^{-2}$. This implies the use of the operator $a_{a}^{j}(1,2)\left(\partial / \partial v_{a}^{j}\right)$, where $a_{a}^{j}(1,2)$ is given by

$$
\overrightarrow{\mathrm{a}}_{a}(1,2)=\frac{e_{a}}{m_{a}} \frac{\epsilon^{A} e_{A}}{x_{a A}{ }^{3}}\left\{\left[1+\frac{1}{2 c^{2}}\left(v_{A}^{2}-v_{a}^{2}-2 \overrightarrow{\mathrm{v}}_{a} \cdot \overrightarrow{\mathrm{v}}_{A}-3 \frac{\left(\overrightarrow{\mathrm{x}}_{a A} \cdot \overrightarrow{\mathrm{v}}_{A}\right)^{2}}{x_{a A}{ }^{2}}\right)\right] \overrightarrow{\mathrm{x}}_{a A}-c^{-2}\left(\overrightarrow{\mathrm{x}}_{a A} \cdot \overrightarrow{\mathrm{v}}_{a}\right)\left(\overrightarrow{\mathrm{v}}_{a}-\overrightarrow{\mathrm{v}}_{A}\right)\right\} .
$$


To find the multipolar expansion, we must now write $\vec{x}_{a}$ as $\vec{X}_{1}+\vec{r}_{a}$ and then use (2.8) and (2.9) to write $\overrightarrow{\mathbf{x}}_{a A} / \overrightarrow{\mathbf{x}}_{a A}^{3}$ up to terms of order $c^{-2}$, that is,

$$
\begin{aligned}
& \frac{\overrightarrow{\mathrm{x}}_{a A}}{x_{a A}{ }^{3}}=\frac{\overrightarrow{\mathrm{R}}}{R^{3}}+\frac{\overrightarrow{\mathrm{r}}_{a}-\overrightarrow{\mathrm{r}}_{A}}{R^{3}}-3 \frac{\overrightarrow{\mathrm{R}} \cdot\left(\overrightarrow{\mathrm{r}}_{a}-\overrightarrow{\mathrm{r}}_{A}\right) \overrightarrow{\mathrm{R}}}{R^{5}}+3 \frac{\overrightarrow{\mathrm{r}}_{a} \cdot \overrightarrow{\mathrm{r}}_{A}}{R^{5}} \overrightarrow{\mathrm{R}}+3 \frac{\overrightarrow{\mathrm{r}}_{a} \cdot \overrightarrow{\mathrm{R}}}{R^{5}} \overrightarrow{\mathrm{r}}_{A}+3 \frac{\overrightarrow{\mathrm{r}}_{A} \cdot \overrightarrow{\mathrm{R}}}{R^{5}} \overrightarrow{\mathrm{r}}_{a}-15 \frac{\left(\overrightarrow{\mathrm{R}} \cdot \overrightarrow{\mathrm{r}}_{a}\right)\left(\overrightarrow{\mathrm{R}} \cdot \overrightarrow{\mathrm{r}}_{A}\right)}{R^{7}} \overrightarrow{\mathrm{R}} \\
& +c^{-2}\left(-\frac{1}{2} \frac{\left(\overrightarrow{\mathrm{r}}_{a} \cdot \overrightarrow{\mathrm{V}}_{1}\right) \overrightarrow{\mathrm{V}}_{1}-\left(\overrightarrow{\mathrm{r}}_{A} \cdot \overrightarrow{\mathrm{V}}_{2}\right) \overrightarrow{\mathrm{V}}_{2}}{R^{3}}-\frac{\left(\overrightarrow{\mathrm{r}}_{a} \cdot \overrightarrow{\mathrm{V}}_{1}\right) \overrightarrow{\mathrm{w}}_{a}-\left(\overrightarrow{\mathrm{r}}_{A} \cdot \overrightarrow{\mathrm{V}}_{2}\right) \overrightarrow{\mathrm{w}}_{A}}{R^{3}}\right. \\
& +\frac{3}{2} \frac{\overrightarrow{\mathrm{R}} \cdot\left[\left(\overrightarrow{\mathrm{r}}_{a} \cdot \overrightarrow{\mathrm{V}}_{1}\right) \overrightarrow{\mathrm{V}}_{1}-\left(\overrightarrow{\mathrm{r}}_{A} \cdot \overrightarrow{\mathrm{V}}_{2}\right) \overrightarrow{\mathrm{V}}_{2}\right]}{R^{5}} \overrightarrow{\mathrm{R}}+3 \frac{\overrightarrow{\mathrm{R}} \cdot\left[\left(\overrightarrow{\mathrm{r}}_{a} \cdot \overrightarrow{\mathrm{V}}_{1}\right) \overrightarrow{\mathrm{w}}_{a}-\left(\overrightarrow{\mathrm{r}}_{A} \cdot \overrightarrow{\mathrm{V}}_{2}\right) \overrightarrow{\mathrm{w}}_{A}\right]}{R^{5}} \overrightarrow{\mathrm{R}} \\
& -\frac{3}{2} \frac{\left(\overrightarrow{\mathrm{r}}_{a} \cdot \overrightarrow{\mathrm{V}}_{2}\right)\left(\overrightarrow{\mathrm{r}}_{A} \cdot \overrightarrow{\mathrm{V}}_{2}\right)}{R^{5}} \overrightarrow{\mathrm{R}}-\frac{3}{2} \frac{\left(\overrightarrow{\mathrm{r}}_{a} \cdot \overrightarrow{\mathrm{V}}_{1}\right)\left(\overrightarrow{\mathrm{r}}_{A} \cdot \overrightarrow{\mathrm{V}}_{1}\right)}{R^{5}} \overrightarrow{\mathrm{R}}-3 \frac{\left(\overrightarrow{\mathrm{r}}_{A} \cdot \overrightarrow{\mathrm{V}}_{2}\right)\left(\overrightarrow{\mathrm{r}}_{a} \cdot \overrightarrow{\mathrm{w}}_{a}\right)}{R^{5}} \overrightarrow{\mathrm{R}} \\
& -3 \frac{\left(\overrightarrow{\mathrm{r}}_{a} \cdot \overrightarrow{\mathrm{V}}_{1}\right)\left(\overrightarrow{\mathrm{r}}_{A} \cdot \overrightarrow{\mathrm{W}}_{a}\right)}{R^{5}} \overrightarrow{\mathrm{R}}-\frac{3}{2} \frac{\left(\overrightarrow{\mathrm{r}}_{a} \cdot \overrightarrow{\mathrm{R}}\right)\left(\overrightarrow{\mathrm{r}}_{A} \cdot \overrightarrow{\mathrm{V}}_{2}\right)}{R^{5}} \overrightarrow{\mathrm{V}}_{2}-\frac{3}{2} \frac{\left(\overrightarrow{\mathrm{r}}_{a} \cdot \overrightarrow{\mathrm{V}}_{1}\right)\left(\overrightarrow{\mathrm{r}}_{A} \cdot \overrightarrow{\mathrm{R}}\right)}{R^{5}} \overrightarrow{\mathrm{V}}_{1} \\
& -3 \frac{\left(\overrightarrow{\mathrm{r}}_{a} \cdot \overrightarrow{\mathrm{R}}\right)\left(\overrightarrow{\mathrm{r}}_{A} \cdot \overrightarrow{\mathrm{V}}_{2}\right)}{R^{5}} \overrightarrow{\mathrm{w}}_{A}-3 \frac{\left(\overrightarrow{\mathrm{r}}_{a} \cdot \overrightarrow{\mathrm{V}}_{1}\right)\left(\overrightarrow{\mathrm{r}}_{A} \cdot \overrightarrow{\mathrm{R}}\right)}{R^{5}} \overrightarrow{\mathrm{w}}_{a}-\frac{3}{2} \frac{\left(\overrightarrow{\mathrm{r}}_{a} \cdot \overrightarrow{\mathrm{V}}_{1}\right)\left(\overrightarrow{\mathrm{V}}_{1} \cdot \overrightarrow{\mathrm{R}}\right)}{R^{5}} \overrightarrow{\mathrm{r}}_{A}-\frac{3}{2} \frac{\left(\overrightarrow{\mathrm{r}}_{A} \cdot \overrightarrow{\mathrm{V}}_{2}\right)\left(\overrightarrow{\mathrm{V}}_{2} \cdot \overrightarrow{\mathrm{R}}\right)}{R^{5}} \overrightarrow{\mathrm{r}}_{a} \\
& -3 \frac{\left(\overrightarrow{\mathrm{r}}_{a} \cdot \overrightarrow{\mathrm{V}}_{1}\right)\left(\overrightarrow{\mathrm{w}}_{a} \cdot \overrightarrow{\mathrm{R}}\right)}{R^{5}} \overrightarrow{\mathrm{r}}_{A}-3 \frac{\left(\overrightarrow{\mathrm{r}}_{a} \cdot \overrightarrow{\mathrm{V}}_{2}\right)\left(\overrightarrow{\mathrm{w}}_{A} \cdot \overrightarrow{\mathrm{R}}\right)}{R^{5}} \overrightarrow{\mathrm{r}}_{a}+\frac{15}{2} \frac{\left(\overrightarrow{\mathrm{R}} \cdot \overrightarrow{\mathrm{r}}_{a}\right)\left[\left(\overrightarrow{\mathrm{r}}_{A} \cdot \overrightarrow{\mathrm{V}}_{2}\right)\left(\overrightarrow{\mathrm{V}}_{2} \cdot \overrightarrow{\mathrm{R}}\right)+2\left(\overrightarrow{\mathrm{r}}_{A} \cdot \overrightarrow{\mathrm{V}}_{2}\right)\left(\overrightarrow{\mathrm{w}}_{A} \cdot \overrightarrow{\mathrm{R}}\right)\right]}{R^{7}} \overrightarrow{\mathrm{R}} \\
& \left.+\frac{15}{2} \frac{\left(\overrightarrow{\mathrm{R}} \cdot \overrightarrow{\mathrm{r}}_{A}\right)\left[\left(\overrightarrow{\mathrm{r}}_{a} \cdot \overrightarrow{\mathrm{V}}_{1}\right)\left(\overrightarrow{\mathrm{V}}_{1} \cdot \overrightarrow{\mathrm{R}}\right)+2\left(\overrightarrow{\mathrm{r}}_{a} \cdot \overrightarrow{\mathrm{V}}_{1}\right)\left(\overrightarrow{\mathrm{w}}_{a} \cdot \overrightarrow{\mathrm{R}}\right)\right]}{R^{7}} \overrightarrow{\mathrm{R}}\right)
\end{aligned}
$$

where $\overrightarrow{\mathbf{R}}=\overrightarrow{\mathrm{X}}_{1}-\overrightarrow{\mathrm{X}}_{2}$.

Let us now compute $\dot{\vec{K}}_{1}$,

$$
\dot{\overrightarrow{\mathrm{K}}}_{1}=a_{a}^{j}(1,2) \frac{\partial \overrightarrow{\mathrm{K}}_{1}}{\partial v_{a}^{j}}=c^{-2} \epsilon^{a} m_{a}\left[\overrightarrow{\mathrm{v}}_{a} \cdot \overrightarrow{\mathrm{a}}_{a}(1,2)\right] \overrightarrow{\mathrm{x}}_{a} \text {. }
$$

From (4.6)

$$
\dot{K}_{1}^{j}=c^{-2}\left[Q_{1} Q_{2} \frac{\overrightarrow{\mathrm{R}} \cdot \overrightarrow{\mathrm{V}}_{1}}{R^{3}} X_{1}^{j}+Q_{2} \frac{M_{1}^{j k} R_{k}}{R^{3}}\right],
$$

where $Q_{1}=\epsilon^{a} e_{a}, Q_{2}=\epsilon^{A} e_{A}$. Using now (2.13) and (2.10)

$$
\dot{\overrightarrow{\mathrm{K}}}_{1}=c^{-2}\left(Q_{1} Q_{2} \frac{\overrightarrow{\mathrm{R}} \cdot \overrightarrow{\mathrm{V}}_{1}}{R^{3}} \overrightarrow{\mathrm{X}}_{1}+\frac{Q_{2}}{2} \frac{\overrightarrow{\mathrm{R}} \times \vec{\mu}_{1}}{R^{3}}\right) \text {. }
$$

For $\overrightarrow{\mathrm{P}}_{1}$ we have

$$
\dot{P}_{1}=\epsilon^{a} \epsilon^{A} \frac{e_{a} e_{A}}{x_{a A}{ }^{3}}\left\{\left[1+\frac{1}{2 c^{2}}\left(v_{A}^{2}-2 \overrightarrow{\mathrm{v}}_{a} \cdot \overrightarrow{\mathrm{v}}_{A}-3 \frac{\left(\overrightarrow{\mathrm{x}}_{a A} \cdot \overrightarrow{\mathrm{v}}_{A}\right)^{2}}{x_{a A}{ }^{2}}\right)\right] \overrightarrow{\mathrm{x}}_{a A}+c^{-2}\left(\overrightarrow{\mathrm{x}}_{a A} \cdot \overrightarrow{\mathrm{v}}_{a}\right) \overrightarrow{\mathrm{v}}_{A}\right\} .
$$

Using (4.6), (2.13), and (2.10) we find, after a straightforward but somewhat long calculation,

$$
\begin{aligned}
\dot{\overrightarrow{\mathrm{P}}}= & \frac{Q_{1} Q_{2}}{R^{3}}\left\{\left[1+\frac{1}{2 c^{2}}\left(V_{2}^{2}-2 \overrightarrow{\mathrm{V}}_{1} \cdot \overrightarrow{\mathrm{V}}_{2}-3 \frac{\left(\overrightarrow{\mathrm{R}} \cdot \overrightarrow{\mathrm{V}}_{2}\right)^{2}}{R^{2}}\right)\right] \overrightarrow{\mathrm{R}}+c^{-2}\left(\overrightarrow{\mathrm{R}} \cdot \overrightarrow{\mathrm{V}}_{1}\right) \overrightarrow{\mathrm{V}}_{2}\right\} \\
+ & c^{-2}\left[-\frac{3}{2} Q_{2} \frac{(\overrightarrow{\mathrm{R}} \times \overrightarrow{\mathrm{V}}) \cdot \vec{\mu}_{1}}{R^{5}} \overrightarrow{\mathrm{R}}-\frac{3}{2} Q_{1} \frac{(\overrightarrow{\mathrm{R}} \times \overrightarrow{\mathrm{V}}) \cdot \vec{\mu}_{2}}{R^{5}} R+\frac{1}{2} Q_{2} \frac{\overrightarrow{\mathrm{V}} \times \vec{\mu}_{1}}{R^{3}}+Q_{1} \frac{\overrightarrow{\mathrm{V}} \times \vec{\mu}_{2}}{R^{3}}-\frac{3}{2} Q_{1}(\overrightarrow{\mathrm{R}} \cdot \overrightarrow{\mathrm{V}}) \frac{\overrightarrow{\mathrm{R}} \times \vec{\mu}_{2}}{R^{5}}\right. \\
& \left.+\frac{9}{4} \frac{\left(\vec{\mu}_{1} \cdot \vec{\mu}_{2}\right)}{R^{5}} \overrightarrow{\mathrm{R}}-\frac{15}{4} \frac{\left(\overrightarrow{\mathrm{R}} \cdot \vec{\mu}_{1}\right)\left(\overrightarrow{\mathrm{R}} \cdot \vec{\mu}_{2}\right)}{R^{7}} \overrightarrow{\mathrm{R}}+\frac{3}{4} \frac{\left(\overrightarrow{\mathrm{R}} \times \vec{\mu}_{1}\right) \times \vec{\mu}_{2}}{R^{5}}+\frac{3}{4} \frac{\left(\overrightarrow{\mathrm{R}} \times \vec{\mu}_{2}\right) \times \vec{\mu}_{1}}{R^{5}}\right],
\end{aligned}
$$


where $\vec{V}=\vec{V}_{1}-\vec{V}_{2}$.

For $\overrightarrow{\mathrm{J}}_{1}$ we have

$\dot{\vec{J}}_{1}=\epsilon^{a} m_{a} \overrightarrow{\mathbf{x}}_{a} \times \overrightarrow{\mathrm{a}}_{a}(1,2)+c^{-2}\left\{\epsilon^{a} \frac{1}{2} m_{a} v_{a}^{2} \overrightarrow{\mathrm{x}}_{a} \times \overrightarrow{\mathrm{a}}_{a}(1,2)\right.$

$$
\left.=\epsilon^{a} \overrightarrow{\mathrm{x}}_{a} \times \overrightarrow{\mathbf{a}}_{a}^{j}(1,2) \frac{\partial \overrightarrow{\mathrm{P}}_{1}}{\partial v_{a}^{j}}, \quad+\epsilon^{a} m_{a}\left[\overrightarrow{\mathrm{v}}_{a} \cdot \overrightarrow{\mathrm{a}}_{a}(1,2)\right] \overrightarrow{\mathrm{x}}_{a} \times \overrightarrow{\mathrm{v}}_{a}\right\}
$$

which gives

$$
\begin{aligned}
\dot{\vec{J}}_{1}=\overrightarrow{\mathrm{X}}_{1} \times \dot{\overrightarrow{\mathrm{P}}}_{1}+c^{-2} \frac{Q_{2}}{R^{3}}\left[\frac{1}{2}\left(\overrightarrow{\mathrm{R}} \cdot \overrightarrow{\mathrm{V}}_{1}\right) \vec{\mu}_{1}-\frac{1}{2}\left(\overrightarrow{\mathrm{R}} \cdot \vec{\mu}_{1}\right) \overrightarrow{\mathrm{V}}_{1}\right. \\
\left.+\frac{1}{2}\left(\overrightarrow{\mathrm{R}} \cdot \vec{\mu}_{1}\right) \overrightarrow{\mathrm{V}}_{2}-\frac{1}{2}\left(\overrightarrow{\mathrm{V}}_{2} \cdot \vec{\mu}_{1}\right) \overrightarrow{\mathrm{R}}\right]+\frac{1}{c^{2} R^{3}} \\
\times\left[\frac{1}{2}\left(\vec{\mu}_{1} \times \vec{\mu}_{2}\right)-\frac{3}{4} \frac{\left(\overrightarrow{\mathrm{R}} \cdot \vec{\mu}_{1}\right)\left(\overrightarrow{\mathrm{R}} \times \vec{\mu}_{2}\right)}{R^{2}}-\frac{3}{4} \frac{\left(\vec{\mu}_{1} \times \vec{\mu}_{2}\right) \cdot \overrightarrow{\mathrm{R}}}{R^{2}} \overrightarrow{\mathrm{R}}\right] .
\end{aligned}
$$

From $H_{1}$ we get

$$
c^{-2} H_{1}=\epsilon^{a} m_{a}+\frac{1}{2 c^{2}} \epsilon^{a} m_{a} v_{a}^{2}+\frac{1}{2 c^{2}} \epsilon^{a} \epsilon^{a^{\prime}} \frac{e_{a} e_{a^{\prime}}}{x_{a a^{\prime}}} .
$$

Therefore,

$$
c^{-2} \dot{H}_{1}=c^{-2} \epsilon^{a} \epsilon^{A} \frac{e_{a} e_{A}}{x_{a A}{ }^{3}} \overrightarrow{\mathrm{x}}_{a A} \cdot \overrightarrow{\mathrm{v}}_{a},
$$

which gives

$$
c^{-2} \dot{H}_{1}=c^{-2} \frac{Q_{1} Q_{2}}{R^{3}} \overrightarrow{\mathrm{R}} \cdot \overrightarrow{\mathrm{V}}_{1} .
$$

Using these quantities we can calculate $V_{1}$ through the relation

$$
\begin{aligned}
\overrightarrow{\mathrm{V}}_{1}=\frac{\overrightarrow{\mathrm{P}}_{1}}{c^{-2} H_{1}}+\frac{\dot{\mathrm{K}}_{1}}{M_{1}}-\frac{1}{M_{1} c^{2}} & {\left[\dot{\mathrm{H}}_{1} \overrightarrow{\mathrm{X}}_{1}+\overrightarrow{\mathrm{V}}_{1} \times \dot{\overrightarrow{\mathrm{J}}}_{1}+\frac{\overrightarrow{\mathrm{P}}_{1} \times \overrightarrow{\mathrm{S}}_{1}}{M_{1}}\right.} \\
& \left.+\left(\overrightarrow{\mathrm{X}}_{1} \cdot \overrightarrow{\mathrm{V}}_{1}\right) \dot{\overrightarrow{\mathrm{P}}}_{1}-\left(\dot{\overrightarrow{\mathrm{P}}}_{1} \cdot \overrightarrow{\mathrm{V}}_{1}\right) \overrightarrow{\mathrm{X}}_{1}\right] .
\end{aligned}
$$

This gives

$$
\overrightarrow{\mathrm{V}}_{1}=\frac{\overrightarrow{\mathrm{P}}_{1}}{c^{-2} H_{1}}+c^{-2} \frac{Q_{2}}{M_{1} R^{3}} \overrightarrow{\mathrm{R}} \times\left[\frac{\vec{\mu}_{1}}{2}-\frac{Q_{1}}{M_{1}} \overrightarrow{\mathrm{S}}_{1}\right] .
$$

To complete the procedure we must calculate $\overrightarrow{\mathrm{K}}_{1}$, which can easily seen to be

$\ddot{\overrightarrow{\mathrm{K}}}_{1}=c^{-2}\left\{Q_{1} Q_{2}\left[\frac{\overrightarrow{\mathrm{R}} \cdot \overrightarrow{\mathrm{V}}_{1}}{R^{3}} \overrightarrow{\mathrm{V}}_{1}+\frac{\overrightarrow{\mathrm{V}} \cdot \overrightarrow{\mathrm{V}}_{1}}{R^{3}} \overrightarrow{\mathrm{R}}-3 \frac{(\overrightarrow{\mathrm{R}} \cdot \overrightarrow{\mathrm{V}})\left(\overrightarrow{\mathrm{R}} \cdot \overrightarrow{\mathrm{V}}_{1}\right)}{R^{5}}\right.\right.$

$$
\left.\left.\times \overrightarrow{\mathrm{R}} \frac{Q_{2}}{2} \frac{\overrightarrow{\mathrm{V}} \times \vec{\mu}_{1}}{R^{3}}-3 \frac{\overrightarrow{\mathrm{R}} \cdot \overrightarrow{\mathrm{V}}}{R^{5}} \overrightarrow{\mathrm{R}} \times \vec{\mu}_{1}\right]\right\},
$$

$c^{-2} \ddot{H}_{1}$ which is given by

$$
c^{-2} \ddot{H}_{1}=c^{-2} Q_{1} Q_{2}\left[\frac{\overrightarrow{\mathrm{V}} \cdot \overrightarrow{\mathrm{V}}_{1}}{R^{3}}-3 \frac{(\overrightarrow{\mathrm{R}} \cdot \overrightarrow{\mathrm{V}})\left(\overrightarrow{\mathrm{R}} \cdot \overrightarrow{\mathrm{V}}_{1}\right)}{R^{5}}\right],
$$

$c^{-2} \ddot{\overrightarrow{\mathrm{P}}}_{1}$ which is given by

$$
c^{-2} \ddot{\overrightarrow{\mathrm{P}}}_{1}=c^{-2} Q_{1} Q_{2}\left[\frac{\overrightarrow{\mathrm{V}}}{R^{3}}-3 \frac{\overrightarrow{\mathrm{R}} \cdot \overrightarrow{\mathrm{V}}}{R^{5}} \overrightarrow{\mathrm{R}}\right],
$$

and $c^{-2} \ddot{\vec{J}}_{1}$ which is given by

$$
c^{-2} \ddot{\vec{J}}_{1}=c^{-2}\left[\overrightarrow{\mathrm{X}}_{1} \times \ddot{\overrightarrow{\mathrm{P}}}_{1}+Q_{1} Q_{2} \frac{\overrightarrow{\mathrm{V}}_{1} \times \overrightarrow{\mathrm{R}}}{R^{3}}\right] .
$$

With these quantities we can now calculate the equations of motion which have the following expressions:

$$
\begin{aligned}
\frac{d M_{1}}{d t}= & 0, \\
\frac{d \overrightarrow{\mathrm{S}}_{1}}{d t}= & -\frac{1}{2 c^{2}} \frac{Q_{1} Q_{2}}{M_{1}} \frac{\left(\overrightarrow{\mathrm{V}}_{1} \times \overrightarrow{\mathrm{R}}\right) \times \overrightarrow{\mathrm{S}}_{1}}{R^{3}}+\frac{1}{2 c^{2}} Q_{2} \frac{(\overrightarrow{\mathrm{V}} \times \overrightarrow{\mathrm{R}}) \times \vec{\mu}_{1}}{R^{3}} \\
& +\frac{1}{c^{2} R^{3}}\left[\frac{1}{2}\left(\vec{\mu}_{1} \times \vec{\mu}_{2}\right)-\frac{3}{4} \frac{\left[\left(\vec{\mu}_{1} \times \vec{\mu}_{2}\right) \cdot \overrightarrow{\mathrm{R}}\right] \overrightarrow{\mathrm{R}}}{R^{2}}\right. \\
& \left.\quad-\frac{3\left(\overrightarrow{\mathrm{R}} \cdot \vec{\mu}_{1}\right)\left(\overrightarrow{\mathrm{R}} \times \vec{\mu}_{2}\right)}{4 R^{2}}\right],
\end{aligned}
$$

and

$$
\begin{aligned}
\frac{d^{2} \overrightarrow{\mathrm{X}}_{1}}{d t^{2}}= & \frac{Q_{1} Q_{2}}{M_{1}} \frac{\overrightarrow{\mathrm{R}}}{R^{3}}+c^{-2} \frac{Q_{1} Q_{2}}{M_{1} R^{3}}\left\{\overrightarrow{\mathrm{R}}\left[\frac{V_{2}^{2}}{2}-\frac{V_{1}{ }^{2}}{2}-\overrightarrow{\mathrm{V}}_{1} \cdot \overrightarrow{\mathrm{V}}_{2}-\frac{3}{2} \frac{\left(\overrightarrow{\mathrm{R}} \cdot \overrightarrow{\mathrm{V}}_{2}\right)^{2}}{R^{2}}\right]-(\overrightarrow{\mathrm{R}} \cdot \overrightarrow{\mathrm{V}})\right) \\
& +\frac{Q_{1}}{c^{2} M_{1} R^{3}}\left[\overrightarrow{\mathrm{V}} \times \vec{\mu}_{2}-\frac{3}{2}(\overrightarrow{\mathrm{R}} \cdot \overrightarrow{\mathrm{V}}) \frac{\left(\overrightarrow{\mathrm{R}} \times \mu_{2}\right)}{R^{2}}-\frac{3}{2} \frac{(\overrightarrow{\mathrm{R}} \times \overrightarrow{\mathrm{V}}) \cdot \vec{\mu}_{2}}{R^{2}} \overrightarrow{\mathrm{R}}\right] \\
& +\frac{Q_{2}}{c^{2} M_{1} R^{3}}\left\{\overrightarrow{\mathrm{V}} \times \vec{\mu}_{1}-\frac{Q_{1}}{M_{1}{ }^{1}} \overrightarrow{\mathrm{V}} \times \overrightarrow{\mathrm{S}_{1}}-\frac{3}{2} \frac{(\overrightarrow{\mathrm{R}} \cdot \overrightarrow{\mathrm{V}})}{R^{2}}\left[\left(\overrightarrow{\mathrm{R}} \times \vec{\mu}_{1}\right)-\frac{2 Q_{1}}{M_{1}}\left(\overrightarrow{\mathrm{R}} \times \overrightarrow{\mathrm{S}}_{1}\right)\right]-\frac{3(\overrightarrow{\mathrm{R}} \times \overrightarrow{\mathrm{V}}) \cdot \vec{\mu}_{1}}{R^{2}} \overrightarrow{\mathrm{R}}\right\} \\
& +\frac{3}{c^{2} 4 M_{1} R^{5}}\left[\left(\overrightarrow{\mathrm{R}} \cdot \vec{\mu}_{1}\right) \vec{\mu}_{2}+\left(\overrightarrow{\mathrm{R}} \cdot \vec{\mu}_{2}\right) \vec{\mu}_{1}+\left(\vec{\mu}_{1} \cdot \overrightarrow{\mu_{2}}\right) \overrightarrow{\mathrm{R}}-\frac{5\left(\overrightarrow{\mathrm{R}} \cdot \vec{\mu}_{1}\right)\left(\overrightarrow{\mathrm{R}} \cdot \vec{\mu}_{2}\right)}{R^{2}} \overrightarrow{\mathrm{R}}\right] .
\end{aligned}
$$

If now we assume $\vec{\mu}_{i}=g_{i}\left(Q_{i} / M_{i}\right) \overrightarrow{\mathrm{S}}_{i}$ this system becomes closed. As can easily be seen, Eq. (4.18) agrees with the Bargmann-Michel-Telegdi equation. ${ }^{6}$

\section{GRAVITATIONAL INTERACTION}

Using the Einstein-Infeld-Hoffmann Lagrangian in the same way we have used the Darwin Lagran- 
gian in the preceding section, we shall construct a set of dynamical equations which give account of the gravitational interaction among bodies of finite size up to order $c^{-2}$. As the hypotheses and procedure will be the same used before for the electromagnetic interaction we shall not insist on them again. The calculation of $H_{i}, \overrightarrow{\mathrm{P}}_{i}, \overrightarrow{\mathrm{J}}_{i}$, and $\overrightarrow{\mathrm{K}}_{i}$ gives

$$
\begin{aligned}
H_{1}= & \epsilon^{a} m_{a} c^{2}+\epsilon^{a} \frac{1}{2} m_{a} v_{a}^{2}-\frac{G}{2} \epsilon^{a} \epsilon^{a^{\prime}} \frac{m_{a} m_{a^{\prime}}}{x_{a a^{\prime}}} \\
+ & c^{-2}\left\{\epsilon^{a} m_{a} \frac{3}{8} v_{a}^{2}+G \epsilon^{a} \epsilon^{a^{\prime}} \frac{m_{a} m_{a^{\prime}}}{x_{a a^{\prime}}}\left[\frac{3}{2} v_{a}^{2}-\frac{7}{4}\left(\overrightarrow{\mathrm{v}}_{a} \cdot \overrightarrow{\mathrm{v}}_{a^{\prime}}\right)\right.\right. \\
& \left.\left.-\frac{1}{4} \frac{\left(x_{a a^{\prime}} \cdot \overrightarrow{\mathrm{v}}_{a}\right)\left(\overrightarrow{\mathrm{x}}_{a a^{\prime}} \cdot \overrightarrow{\mathrm{v}}_{a^{\prime}}\right)}{x_{a a^{\prime}}{ }^{\prime}}\right]\right\} \\
\overrightarrow{\mathrm{P}}_{1}= & \epsilon^{a} m_{a} \overrightarrow{\mathrm{v}}_{a}\left[1+c^{-2}\left(\frac{v_{a}^{2}}{2}-\frac{G}{2} \frac{\epsilon^{a^{\prime}} m_{a^{\prime}}}{x_{a a^{\prime}}}\right)\right] \\
& -\frac{G}{2 c^{2}} \epsilon^{a} \epsilon^{a{ }^{\prime}} \frac{m_{a} m_{a^{\prime}}}{x_{a a^{\prime}}{ }^{3}}\left(\overrightarrow{\mathrm{x}}_{a a^{\prime}} \cdot \overrightarrow{\mathrm{v}}_{a^{\prime}}\right) \overrightarrow{\mathrm{X}}_{a a^{\prime}},
\end{aligned}
$$

$\vec{J}_{1}=\epsilon^{a} m_{a} \overrightarrow{\mathrm{x}}_{a} \times \overrightarrow{\mathrm{v}}_{a}$

$$
\begin{array}{r}
+c^{-2}\left\{\epsilon^{a} m_{a} \frac{v_{a}^{2}}{2} \overrightarrow{\mathrm{x}}_{a} \times \overrightarrow{\mathrm{v}}_{a}+G \epsilon^{a} \epsilon^{a^{\prime}} \frac{m_{a} m_{a^{\prime}}}{x_{a a^{\prime}}} \overrightarrow{\mathrm{x}}_{a}\right. \\
\left.\times\left[3 \overrightarrow{\mathrm{v}}_{a}-\frac{7}{2} \overrightarrow{\mathrm{v}}_{a^{\prime}}-\frac{1}{2} \frac{\left(\overrightarrow{\mathrm{x}}_{a a^{\prime}} \cdot \overrightarrow{\mathrm{v}}_{a^{\prime}}\right) \overrightarrow{\mathrm{x}}_{a a^{\prime}}}{x_{a a^{\prime}}{ }^{2}}\right]\right\}, \\
\overrightarrow{\mathrm{K}}_{1}=\epsilon^{a} m_{a} \overrightarrow{\mathrm{x}}_{a}+c^{-2}\left[\epsilon^{a} m_{a} \overrightarrow{\mathrm{x}}_{a}\left(\frac{v_{a}^{2}}{2}-\frac{G \epsilon^{a^{\prime}} m_{a^{\prime}}}{2}\right)\right] .
\end{array}
$$

To compute now the functions $\dot{\Lambda}$ we need $\vec{a}_{a}(1,2)$ which is given by

$$
\begin{aligned}
& \overrightarrow{\mathrm{a}}_{a}(1,2)=-G \frac{\epsilon^{A} m_{A} \overrightarrow{\mathrm{x}}_{a A}}{x_{a A}{ }^{3}} \\
&+G \frac{\epsilon^{A} m_{A}}{c^{2} x_{a A}{ }^{3}}\left\{\left[-v_{a}^{2}-2 v_{A}^{2}+4 \overrightarrow{\mathrm{v}}_{a} \cdot \overrightarrow{\mathrm{v}}_{A}\right.\right. \\
&\left.+\frac{3}{2} \frac{\left(\overrightarrow{\mathrm{v}}_{A} \cdot \overrightarrow{\mathrm{x}}_{a A}\right)^{2}}{x_{a A}{ }^{2}}\right] \overrightarrow{\mathrm{x}}_{a A} \\
&\left.+\left(\overrightarrow{\mathrm{v}}_{a}-\overrightarrow{\mathrm{v}}_{A}\right)\left[\left(4 \overrightarrow{\mathrm{v}}_{a}-3 \overrightarrow{\mathrm{v}}_{A}\right) \cdot \overrightarrow{\mathrm{x}}_{a A}\right]\right\} .
\end{aligned}
$$

Here as the $\overrightarrow{\mathrm{x}}_{a A}$ dependence of the Newtonian part of (5.5) is the same as that of the Coulombian force we can use again (4.6).

We find now

$$
\dot{\overrightarrow{\mathrm{K}}}_{1}=c^{-2} \epsilon^{a} m_{a}\left[\overrightarrow{\mathrm{v}}_{a} \cdot \overrightarrow{\mathrm{a}}_{a}(1,2)\right] \overrightarrow{\mathrm{x}}_{a},
$$

and, as for the gravitational interaction

$$
\overrightarrow{\mathrm{S}}_{i}=\vec{\mu}_{i}+O\left(c^{-2}\right)
$$

we have

$$
\dot{\overrightarrow{\mathrm{K}}}_{1}=-\frac{G}{c^{2}}\left(M_{1} M_{2} \frac{\overrightarrow{\mathrm{R}} \cdot \overrightarrow{\mathrm{V}}_{1}}{R^{3}} \overrightarrow{\mathrm{X}}+\frac{M_{2}}{2} \frac{\overrightarrow{\mathrm{R}} \times \overrightarrow{\mathrm{S}}_{1}}{R^{3}}\right) .
$$

For $\dot{\overrightarrow{\mathrm{P}}}_{1}$ we must compute

$$
\begin{aligned}
\overrightarrow{\mathrm{P}}_{1}=\epsilon^{a} m_{a} \overrightarrow{\mathrm{a}}_{a}(1,2)+c^{-2}\left[m_{a} \overrightarrow{\mathrm{v}}_{a} \cdot \overrightarrow{\mathrm{a}}_{a}(1,2) \overrightarrow{\mathrm{v}}_{a}\right. \\
+ \\
\left.+\frac{1}{2} m_{a} v_{a}^{2} \overrightarrow{\mathrm{a}}_{a}(1,2)\right] \\
-\frac{G}{2 c^{2}} m_{a} \frac{\epsilon^{a^{\prime}} m_{a^{\prime}}}{x_{a a^{a}}} \overrightarrow{\mathrm{a}}_{a}(1,2),
\end{aligned}
$$

which gives

$$
\begin{aligned}
\dot{\mathrm{P}}_{1}=-\frac{G M_{1} M_{2}}{R^{3}} \overrightarrow{\mathrm{R}}+G c^{-2} & \frac{M_{1} M_{2}}{R^{3}}\left[\left(-\frac{3}{2} V_{1}^{2}-2 V_{2}^{2}+4 \overrightarrow{\mathrm{V}}_{1} \cdot \overrightarrow{\mathrm{V}}_{2}+\frac{3}{2} \frac{\left(\overrightarrow{\mathrm{R}} \cdot \overrightarrow{\mathrm{V}}_{2}\right)^{2}}{R^{2}}\right) \overrightarrow{\mathrm{R}}+3(\overrightarrow{\mathrm{R}} \cdot \overrightarrow{\mathrm{V}}) \overrightarrow{\mathrm{V}}-\left(\overrightarrow{\mathrm{R}} \cdot \overrightarrow{\mathrm{V}}_{1}\right) \overrightarrow{\mathrm{V}}_{2}\right] \\
& -4 M_{1} \frac{\overrightarrow{\mathrm{V}}_{2} \times \overrightarrow{\mathrm{S}}_{2}}{R^{3}}-\frac{7}{2} M_{2} \frac{\overrightarrow{\mathrm{V}} \times \overrightarrow{\mathrm{S}}_{1}}{R^{3}}+6 M_{1} \frac{\left[\left(\overrightarrow{\mathrm{R}} \times(\overrightarrow{\mathrm{V}}) \cdot \overrightarrow{\mathrm{S}}_{2}\right]\right.}{R^{5}} \overrightarrow{\mathrm{R}}+6 M_{2} \frac{\left[(\overrightarrow{\mathrm{R}} \times \overrightarrow{\mathrm{V}}) \cdot \overrightarrow{\mathrm{S}}_{1}\right]}{R^{5}} \overrightarrow{\mathrm{R}}+6 M_{1} \frac{(\overrightarrow{\mathrm{V}} \cdot \overrightarrow{\mathrm{R}})\left(\overrightarrow{\mathrm{R}} \times \overrightarrow{\mathrm{S}}_{2}\right)}{R^{5}} \\
& \left.+\frac{9}{2} M_{2} \frac{(\overrightarrow{\mathrm{V}} \cdot \overrightarrow{\mathrm{R}})\left(\overrightarrow{\mathrm{R}} \times \overrightarrow{\mathrm{S}}_{1}\right)}{R^{5}}-3 \frac{\overrightarrow{\mathrm{S}}_{1} \cdot \overrightarrow{\mathrm{S}}_{2}}{R^{5}} \overrightarrow{\mathrm{R}}-3 \frac{\left(\overrightarrow{\mathrm{R}} \cdot \overrightarrow{\mathrm{S}}_{1}\right) \overrightarrow{\mathrm{S}}_{2}}{R^{5}}-3 \frac{\left(\overrightarrow{\mathrm{R}} \cdot \overrightarrow{\mathrm{S}}_{2}\right) \overrightarrow{\mathrm{S}}_{1}}{R^{5}}+15 \frac{\left(\overrightarrow{\mathrm{S}}_{1} \cdot \overrightarrow{\mathrm{R}}\right)\left(\overrightarrow{\mathrm{S}}_{2} \cdot \overrightarrow{\mathrm{R}}\right)}{R^{7}} \overrightarrow{\mathrm{R}}\right\} .(5.10)
\end{aligned}
$$

For $\dot{\vec{J}}_{1}$ we find the expression

$$
\begin{aligned}
\dot{\mathrm{J}}_{1}=\overrightarrow{\mathrm{X}}_{1} \times \dot{\overrightarrow{\mathrm{P}}}_{1}+G c^{-2} & \left(3 M_{2} \frac{(\overrightarrow{\mathrm{V}} \cdot \overrightarrow{\mathrm{R}}) \overrightarrow{\mathrm{S}}_{1}}{R^{3}}-2 M_{2} \frac{\left(\overrightarrow{\mathrm{V}} \times \overrightarrow{\mathrm{S}}_{1}\right) \times \overrightarrow{\mathrm{R}}}{R^{3}}-\frac{M_{2}}{2} \frac{\left(\overrightarrow{\mathrm{R}} \times \overrightarrow{\mathrm{S}}_{1}\right) \times \overrightarrow{\mathrm{V}}_{2}}{R^{3}}+\frac{3}{2} M_{2} \frac{\left(\overrightarrow{\mathrm{R}} \times \overrightarrow{\mathrm{S}}_{1}\right) \times \overrightarrow{\mathrm{V}}}{R^{3}}-2 \frac{\overrightarrow{\mathrm{S}}_{1} \times \overrightarrow{\mathrm{S}}_{2}}{R^{3}}\right. \\
& \left.+3 \frac{\left(\left(\overrightarrow{\mathrm{S}}_{1} \times \overrightarrow{\mathrm{S}}_{2}\right) \cdot \overrightarrow{\mathrm{R}}\right]}{R^{5}} \overrightarrow{\mathrm{R}}+3 \frac{\left(\overrightarrow{\mathrm{R}} \cdot \overrightarrow{\mathrm{S}}_{1}\right)\left(\overrightarrow{\mathrm{R}} \times \overrightarrow{\mathrm{S}}_{2}\right)}{R^{5}}\right) .
\end{aligned}
$$

And for $c^{-2} H_{1}$ we have

$$
c^{-2} H_{1}=\epsilon^{a} m_{a}+\frac{1}{2 c^{2}} \epsilon^{a} m_{a} v_{a}^{2}-\frac{1}{2 c^{2}} G \epsilon^{a} \epsilon^{a^{\prime}} \frac{m_{a} m_{a^{\prime}}}{x_{a a^{\prime}}} .
$$

Therefore,

$$
c^{-2} \dot{H}_{1}=-G c^{-2} \epsilon^{a} \epsilon^{A} \frac{m_{a} m_{A}}{x_{a A}{ }^{3}} \overrightarrow{\mathrm{x}}_{a A} \cdot \overrightarrow{\mathrm{v}}_{a},
$$

which gives 


$$
c^{-2} \dot{H}_{1}=-G c^{-2} M_{1} M_{2} \frac{\overrightarrow{\mathrm{R}} \cdot \overrightarrow{\mathrm{V}}_{1}}{R^{3}}
$$

We can also calculate $\vec{V}_{1}$ using these expressions in (4.11). The result is

$$
\overrightarrow{\mathrm{V}}_{1}=\frac{\overrightarrow{\mathrm{P}}_{1}}{c^{-2} H_{1}}+\frac{G}{2 c^{2}} \frac{M_{2}}{M_{1}} \frac{\overrightarrow{\mathrm{R}} \times \overrightarrow{\mathrm{S}}_{1}}{R^{3}}
$$

To find the dynamical equations we must calculate the $\ddot{\Lambda}$ functions. We have

$$
\begin{aligned}
& \ddot{\overrightarrow{\mathrm{K}}}_{1}=-G c^{-2}\left\{M_{1} M_{2}\left[\frac{\overrightarrow{\mathrm{R}} \cdot \overrightarrow{\mathrm{V}}_{1}}{R^{3}} \overrightarrow{\mathrm{V}}_{1}+\frac{\overrightarrow{\mathrm{V}} \cdot \overrightarrow{\mathrm{V}}_{1}}{R^{3}} \overrightarrow{\mathrm{X}}_{1}-3 \frac{(\overrightarrow{\mathrm{R}} \cdot \overrightarrow{\mathrm{V}})\left(\overrightarrow{\mathrm{R}} \cdot \overrightarrow{\mathrm{V}}_{1}\right)}{R^{5}} \overrightarrow{\mathrm{X}}_{1}\right]+\frac{\left.M_{2}\left[\frac{\overrightarrow{\mathrm{V}} \times \overrightarrow{\mathrm{S}}_{1}}{2}+3 \frac{(\overrightarrow{\mathrm{R}} \cdot \overrightarrow{\mathrm{V}})}{R^{5}} \overrightarrow{\mathrm{V}} \times \overrightarrow{\mathrm{S}}{ }_{1}\right]\right\},}{c^{-2} \ddot{H}_{1}=-G c^{-2} M_{1} M_{2}\left[\frac{\overrightarrow{\mathrm{V}} \cdot \overrightarrow{\mathrm{V}}_{1}}{R^{3}}-3 \frac{(\overrightarrow{\mathrm{R}} \cdot \overrightarrow{\mathrm{V}})\left(\overrightarrow{\mathrm{R}} \cdot \overrightarrow{\mathrm{V}}_{1}\right)}{R^{5}}\right],}\right. \\
& c^{-2} \ddot{\overrightarrow{\mathrm{P}}}_{1}=-G c^{-2} M_{1} M_{2}\left[\frac{\overrightarrow{\mathrm{V}}}{R^{3}}-3 \frac{\overrightarrow{\mathrm{R}} \cdot \overrightarrow{\mathrm{V}}}{R^{3}} \overrightarrow{\mathrm{R}}\right], \\
& c^{-2} \ddot{\overrightarrow{\mathrm{J}}}_{1}=c^{-2}\left[\overrightarrow{\mathrm{X}}_{1} \times \overrightarrow{\mathrm{P}}_{1}-G M_{1} M_{2} \frac{\overrightarrow{\mathrm{V}}_{1} \times \overrightarrow{\mathrm{R}}}{R^{3}}\right] .
\end{aligned}
$$

Using these functions the equations of motion can easily be written down. The result is

$$
\begin{aligned}
& \frac{d M_{1}}{d t}=0 \\
& \frac{d \overrightarrow{\mathrm{S}}_{1}}{d t}=\frac{G}{c^{2} R^{3}}\left\{3 M_{2}(\overrightarrow{\mathrm{V}} \cdot \overrightarrow{\mathrm{R}}) \overrightarrow{\mathrm{S}}_{1}+\left[2 S_{2}+\frac{M_{2}}{2} \overrightarrow{\mathrm{V}}_{1} \times \overrightarrow{\mathrm{R}}+2 M_{2} \overrightarrow{\mathrm{R}} \times \overrightarrow{\mathrm{V}}+3 \frac{\overrightarrow{\mathrm{R}} \times\left(\overrightarrow{\mathrm{R}} \times \overrightarrow{\mathrm{S}}_{2}\right)}{R^{2}}\right] \times \overrightarrow{\mathrm{S}}_{1}\right\}, \\
& \frac{d^{2} \overrightarrow{\mathrm{X}}_{1}}{d t^{2}}=-G M_{2} \frac{\overrightarrow{\mathrm{R}}}{R^{3}}+G c^{-2}\left\{\frac{M_{2}}{R^{3}}\left[\left(-V_{1}{ }^{2}-2 V_{2}^{2}+4 V_{1} \cdot V_{2}+\frac{3}{2} \frac{\left(\overrightarrow{\mathrm{R}} \cdot \overrightarrow{\mathrm{V}}_{2}\right)^{2}}{R^{2}}\right) \overrightarrow{\mathrm{R}}+\overrightarrow{\mathrm{V}}\left[\left(4 \overrightarrow{\mathrm{V}}_{1}-3 \overrightarrow{\mathrm{V}}_{2}\right) \cdot \overrightarrow{\mathrm{R}}\right]\right]-4 \frac{\overrightarrow{\mathrm{V}} \times \overrightarrow{\mathrm{S}}_{2}}{R^{3}}\right. \\
& -3 \frac{M_{2}}{M_{1}} \frac{\overrightarrow{\mathrm{V}} \times \overrightarrow{\mathrm{S}}_{1}}{R^{3}}+3 \frac{M_{2}}{M_{1}} \frac{\left(\overrightarrow{\mathrm{R}} \cdot \overrightarrow{\mathrm{V}}_{1}\right)\left(\overrightarrow{\mathrm{R}} \times \overrightarrow{\mathrm{S}}_{1}\right)}{R^{5}}+6 \frac{\left[(\overrightarrow{\mathrm{R}} \times \overrightarrow{\mathrm{V}}) \cdot \overrightarrow{\mathrm{S}}_{2}\right] \overrightarrow{\mathrm{R}}}{R^{5}}+6 \frac{M_{2}}{M_{1}} \frac{\left[(\overrightarrow{\mathrm{R}} \times \overrightarrow{\mathrm{V}}) \cdot \overrightarrow{\mathrm{S}_{1}}\right] \overrightarrow{\mathrm{R}}}{R^{5}} \\
& \left.+6 \frac{(\overrightarrow{\mathrm{V}} \cdot \overrightarrow{\mathrm{R}})\left(\overrightarrow{\mathrm{R}} \times \overrightarrow{\mathrm{S}}_{2}\right)}{R^{5}}-3 \frac{\overrightarrow{\mathrm{S}}_{1} \cdot \overrightarrow{\mathrm{S}}_{2}}{M_{1} R^{5}} \overrightarrow{\mathrm{R}}-3 \frac{\left(\overrightarrow{\mathrm{R}} \cdot \overrightarrow{\mathrm{S}}_{1}\right) \overrightarrow{\mathrm{S}}_{2}}{M_{1} R^{5}}-3 \frac{\left(\overrightarrow{\mathrm{R}} \cdot \overrightarrow{\mathrm{S}}_{2}\right) \overrightarrow{\mathrm{S}}_{1}}{M_{1} R^{5}}+15 \frac{\left(\overrightarrow{\mathrm{R}} \cdot \overrightarrow{\mathrm{S}}_{1}\right)\left(\overrightarrow{\mathrm{R}} \cdot \overrightarrow{\mathrm{S}}_{2}\right)}{M_{1} R^{7}} \overrightarrow{\mathrm{R}}\right\} \text {. }
\end{aligned}
$$

These equations can be compared with those given by Pirani, ${ }^{4}$ Corinaldesi and Papapetrou, ${ }^{7}$ and Barker and O'Connell. ${ }^{3}$ As can easily be seen, they reduce to the Pirani equations in the limit $V_{2}=0$.

The equation for the spin is, however, different from those of Cho and Hari Dass ${ }^{2}$ and of Barker and $\mathrm{O}^{\prime}$ Connell. ${ }^{3}$ The spin-precession term is identical with the term given by these authors, but we find a new term $-3 M_{2} G c^{2}(\vec{V} \cdot \vec{R}) \vec{S}_{1} / R^{3}$ proportional to the spin that is responsible for a change in the magnitude of the spin.

The spin vector we have used was chosen attending to its transformation properties. A change in its definition in order to construct a new spin vector with constant magnitude, cannot be made without altering its transformation properties, and must therefore be discarded. We shall comment on this in the conclusions.

\section{LAGRANGIAN FORM OF THE EQUATIONS OF MOTION}

We shall now investigate the possibility of deriving Eqs. (4.19) and (5.22) from a Lagrangian.

The expression (4.19) for the acceleration of the center of mass of a charged body can be divided in two parts: one that does not contain the magnetic moments, which can be derived from the Darwin Lagrangian (hereafter abbreviated as $\mathscr{L}_{D W}$ ), and another that does contain the magnetic moments. Multiplying (4.19) by $M_{1}$ we can write it in the form 


$$
\begin{aligned}
\frac{d}{d t}\left(\overrightarrow{\mathrm{P}}_{1 \mathrm{DW}}\right)= & \overrightarrow{\mathrm{F}}_{1 \mathrm{DW}}+c^{-2} \frac{\overrightarrow{\mathrm{V}}}{R} \times\left(Q_{1} \vec{\mu}_{2}+Q_{2} \vec{\mu}_{1}\right)-\frac{3}{2 c^{2}} \frac{\overrightarrow{\mathrm{R}} \cdot \overrightarrow{\mathrm{V}}}{R^{5}} \overrightarrow{\mathrm{R}} \times\left(Q_{1} \vec{\mu}_{2}+Q_{2} \vec{\mu}_{1}\right)+\frac{3}{2 c^{2}} \frac{\overrightarrow{\mathrm{V}} \cdot\left[\overrightarrow{\mathrm{R}} \times\left(\overrightarrow{\mathrm{Q}}_{1} \vec{\mu}_{2}+\overrightarrow{\mathrm{Q}}_{2} \vec{\mu}_{1}\right)\right]}{R^{5}} \overrightarrow{\mathrm{R}} \\
& -c^{-2} \frac{Q_{1} Q_{2}}{M_{1}} \frac{\overrightarrow{\mathrm{V}} \times \overrightarrow{\mathrm{S}}_{1}}{R^{3}}+\frac{3}{c^{2}} \frac{Q_{1} Q_{2}}{M_{1}} \frac{\overrightarrow{\mathrm{R}} \cdot \overrightarrow{\mathrm{V}}}{R^{5}} \overrightarrow{\mathrm{R}} \times \overrightarrow{\mathrm{S}}_{1} \\
& +\frac{3}{4 c^{2} R^{5}}\left[\left(\overrightarrow{\mathrm{R}} \cdot \vec{\mu}_{1}\right) \vec{\mu}_{2}+\left(\overrightarrow{\mathrm{R}} \cdot \vec{\mu}_{2}\right) \vec{\mu}_{1}+\left(\vec{\mu}_{1} \cdot \vec{\mu}_{2}\right) \overrightarrow{\mathrm{R}}-5 \frac{\left(\overrightarrow{\mathrm{R}} \cdot \vec{\mu}_{1}\right)\left(\overrightarrow{\mathrm{R}} \cdot \vec{\mu}_{2}\right)}{R^{2}} \overrightarrow{\mathrm{R}}\right],
\end{aligned}
$$

where

$$
\overrightarrow{\mathrm{P}}_{1 \mathrm{DW}}=\frac{\partial \mathcal{L}_{\mathrm{DW}}}{\partial \overrightarrow{\mathrm{V}}_{1}} \text { and } \overrightarrow{\mathrm{F}}_{1 \mathrm{DW}}=\frac{\partial \mathcal{L}_{\mathrm{DW}}}{\partial \overrightarrow{\mathrm{X}}_{1}} \text {. }
$$

An easy calculation then proves that

$$
\begin{aligned}
& \frac{1}{4 c^{2}} \vec{\nabla}\left[\frac{\partial}{\partial R^{r}} \frac{\partial}{\partial R^{s}}\left(\frac{1}{R}\right)\right] \mu_{1}^{r} \mu_{2}^{s}=\frac{3}{4 c^{2} R^{5}}\left[\left(\vec{\mu}_{1} \cdot \vec{\mu}_{2}\right) \overrightarrow{\mathrm{R}}+\left(\overrightarrow{\mathrm{R}} \cdot \vec{\mu}_{2}\right) \vec{\mu}_{1}+\left(\overrightarrow{\mathrm{R}} \cdot \vec{\mu}_{1}\right) \vec{\mu}_{2}-5 \frac{\left(\overrightarrow{\mathrm{R}} \cdot \vec{\mu}_{1}\right)\left(\overrightarrow{\mathrm{R}} \cdot \vec{\mu}_{2}\right)}{R^{2}} \overrightarrow{\mathrm{R}}\right], \\
& \frac{d}{d t}\left[\frac{1}{2 c^{2}} \frac{\overrightarrow{\mathrm{R}}}{R^{3}} \times\left(Q_{1} \vec{\mu}_{2}+Q_{2} \vec{\mu}_{1}\right)\right]=\frac{1}{2 c^{2}} \frac{\overrightarrow{\mathrm{V}}}{R^{3}} \times\left(Q_{1} \vec{\mu}_{2}+Q_{2} \vec{\mu}_{1}\right)-\frac{3}{2 c^{2}}(\overrightarrow{\mathrm{R}} \cdot \overrightarrow{\mathrm{V}}) \frac{\overrightarrow{\mathrm{R}}}{R^{5}} \times\left(Q_{1} \vec{\mu}_{2}+Q_{2} \vec{\mu}_{1}\right), \\
& -\vec{\nabla}\left(\frac{1}{2 c^{2}} \frac{\overrightarrow{\mathrm{V}}}{R^{3}} \cdot\left[\overrightarrow{\mathrm{R}} \times\left(Q_{1} \vec{\mu}_{2}+Q_{2} \vec{\mu}_{1}\right)\right]\right)=\frac{1}{2 c^{2}} \frac{\overrightarrow{\mathrm{V}}}{R^{3}} \times\left(Q_{1} \vec{\mu}_{2}+Q_{2} \vec{\mu}_{1}\right)+\frac{3}{2 c^{2}} \frac{\overrightarrow{\mathrm{V}} \cdot\left[\overrightarrow{\mathrm{R}} \times\left(Q_{1} \vec{\mu}_{2}+Q_{2} \vec{\mu}_{1}\right)\right]}{R^{5}} \overrightarrow{\mathrm{R}},
\end{aligned}
$$

and

$$
\frac{d}{d t}\left(\frac{1}{c^{2}} \frac{Q_{1} Q_{2}}{M_{1}} \frac{\overrightarrow{\mathrm{R}} \times \overrightarrow{\mathrm{S}}_{1}}{R^{3}}\right)=\frac{1}{c^{2}} \frac{Q_{1} Q_{2}}{M_{1}} \frac{\overrightarrow{\mathrm{V}} \times \overrightarrow{\mathrm{S}}_{1}}{R^{3}}-\frac{3}{c^{2}} \frac{Q_{1} Q_{2}}{M_{1}}(\overrightarrow{\mathrm{R}} \cdot \overrightarrow{\mathrm{V}}) \frac{\overrightarrow{\mathrm{R}} \times \overrightarrow{\mathrm{S}}_{1}}{R^{5}} .
$$

Using these expressions (6.1) can be written in the form

$$
\begin{aligned}
\frac{d}{d t}\left(\overrightarrow{\mathrm{P}}_{1 \mathrm{DW}}-\frac{1}{2 c^{2}} \frac{\overrightarrow{\mathrm{R}}}{R^{3}} \times\left(Q_{1} \vec{\mu}_{2}+Q_{2} \vec{\mu}_{1}\right)+\frac{1}{c^{2}} \frac{Q_{1} Q_{2}}{M_{1}} \frac{\overrightarrow{\mathrm{R}} \times \overrightarrow{\mathrm{S}}_{1}}{R^{3}}\right)=\overrightarrow{\mathrm{F}}_{1 \mathrm{DW}}+\vec{\nabla}\left\{-\frac{1}{2 c^{2}} \frac{\overrightarrow{\mathrm{V}} \cdot\left[\overrightarrow{\mathrm{R}} \times\left(Q_{1} \vec{\mu}_{2}+Q_{2} \vec{\mu}_{1}\right)\right]}{R^{3}}\right. \\
\left.+\frac{1}{4 c^{2}}\left[\frac{\partial}{\partial R^{r}} \frac{\partial}{\partial R^{s}}\left(\frac{1}{R}\right) \mu_{1}^{r} \mu_{2}^{s}\right]\right\} .
\end{aligned}
$$

Defining now a function $L$ as

$$
L=\&_{\mathrm{DW}}-\frac{1}{2 c^{2}} \frac{\overrightarrow{\mathrm{V}} \cdot\left[\overrightarrow{\mathrm{R}} \times\left(Q_{1} \vec{\mu}_{2}+Q_{2} \vec{\mu}_{1}\right)\right]}{R^{3}}+\frac{1}{4 c^{2}}\left[\frac{\partial}{\partial R^{r}} \frac{\partial}{\partial R^{s}}\left(\frac{1}{R}\right) \mu_{1}^{r} \mu_{2}^{s}\right],
$$

(6.6) can be written in the form

$$
\frac{d}{d t}\left(\frac{\partial L}{\partial \overrightarrow{\mathrm{V}}_{1}}+\frac{1}{c^{2}} \frac{Q_{1} Q_{2}}{M_{1}} \frac{\overrightarrow{\mathrm{R}} \times \overrightarrow{\mathrm{S}}_{1}}{R^{3}}\right)=\frac{\partial L}{\partial \overrightarrow{\mathrm{X}}_{1}} .
$$

Therefore it is manifestly evident that (6.1) cannot be written in Lagrangian form. Let us now try a change of coordinates in order to see if in the new coordinates the equation of motion can be put in Lagrangian form.

We try a transformation of the type

$$
\overrightarrow{\mathrm{X}}_{1}=\overrightarrow{\mathrm{Z}}_{1}+\frac{a}{2 c^{2}} \frac{\overrightarrow{\mathrm{V}}_{1} \times \overrightarrow{\mathrm{S}}_{1}}{M_{1}}
$$

where $a$ is a free dimensionless parameter. If these coordinates are used the changes will only affect the time derivative of the velocity and the
Coulomb force term, that is, the Newtonian terms. Ther efore if a Lagrangian exists in this coordinate system it must be possible to put the expression

$$
\frac{d}{d t}\left(m_{1} \delta \overrightarrow{\mathrm{V}}_{1}+\frac{1}{c^{2}} \frac{Q_{1} Q_{2}}{M_{1}} \frac{\overrightarrow{\mathrm{R}} \times \vec{S}_{1}}{R^{3}}\right)=\delta \text { (Coulomb) }
$$

in Lagrangian form.

From (6.7),

$$
\overrightarrow{\mathrm{V}}_{1}=\dot{\overrightarrow{\mathrm{Z}}}_{1}+\frac{a}{2 c^{2}} \frac{Q_{1} Q_{2}}{M_{1}} \frac{\overrightarrow{\mathrm{Z}} \times \overrightarrow{\mathrm{S}}_{1}}{Z^{3}} \text {. }
$$

Therefore the left-hand side of (6.8) has the form

$$
\frac{d}{d t}\left[\left(\frac{a}{2}+1\right) \frac{Q_{1} Q_{2}}{c^{2} M_{1}} \frac{\overrightarrow{\mathrm{Z}} \times \overrightarrow{\mathrm{S}}_{1}}{Z^{3}}\right] \text {. }
$$

The change in the Coulomb term is given by 


$$
\begin{aligned}
\delta\left(\frac{Q_{1} Q_{2}}{R^{3}} R^{j}\right)=\frac{\partial}{\partial Z^{i}}\left(\frac{Q_{1} Q_{2}}{Z^{3}} Z^{j}\right) \delta Z^{i} & =-\frac{\partial}{\partial Z^{i}} \frac{\partial}{\partial Z^{j}}\left(\frac{Q_{1} Q_{2}}{Z}\right)\left(\frac{a}{2 c^{2}} \frac{\left[\overrightarrow{\mathrm{V}}_{1} \times \overrightarrow{\mathrm{S}}_{1}\right]^{i}}{M_{2}}-\frac{a}{2 c^{2}} \frac{\left[\overrightarrow{\mathrm{V}}_{2} \times \overrightarrow{\mathrm{S}}_{2}\right]^{i}}{M^{2}}\right) \\
& =-\vec{\nabla}^{j}\left[\frac{Q_{1} Q_{2}}{Z^{3}}\left(\frac{a}{2 c^{2}} \frac{\overrightarrow{\mathrm{V}}_{1} \cdot\left(\overrightarrow{\mathrm{Z}} \times \overrightarrow{\mathrm{S}}_{1}\right)}{M_{1}}-\frac{a}{2 c^{2}} \frac{\overrightarrow{\mathrm{V}}_{2} \cdot\left(\overrightarrow{\mathrm{Z}} \times \overrightarrow{\mathrm{S}}_{2}\right)}{M_{2}}\right)\right] .
\end{aligned}
$$

Therefore a Lagrangian exists for $a=-1$, that is,

$$
\overrightarrow{\mathrm{Z}}_{i}=\overrightarrow{\mathrm{X}}_{i}+\frac{1}{2 c^{2}} \frac{\overrightarrow{\mathrm{V}}_{i} \times \overrightarrow{\mathrm{S}}_{i}}{M_{i}}
$$

is a good Lagrangian coordinate. $\overrightarrow{\mathrm{Z}}_{i}$ is precisely the center of spin defined in I.

Using $\vec{\mu}_{i}=g_{i}\left(Q_{i} / M_{i}\right) \overrightarrow{\mathrm{S}}_{i}$, we can write the equation of motion for the $Z$ variable using the Lagrangian

$$
\begin{aligned}
\mathscr{L}= & \mathscr{L}_{\mathrm{DW}}-\frac{1}{2 c^{2}} \frac{Q_{1} Q_{2}}{Z^{3}}\left[\left(g_{1}-1\right) \frac{\overrightarrow{\mathrm{V}}_{1} \cdot\left(\overrightarrow{\mathrm{Z}} \times \overrightarrow{\mathrm{S}}_{1}\right)}{M_{1}}-\left(g_{2}-1\right) \frac{\overrightarrow{\mathrm{V}}_{2} \cdot\left(\overrightarrow{\mathrm{Z}} \times \overrightarrow{\mathrm{S}}_{2}\right)}{M_{2}}+\frac{g_{2}}{M_{2}} \overrightarrow{\mathrm{V}}_{1} \cdot\left(\overrightarrow{\mathrm{Z}} \times \overrightarrow{\mathrm{S}}_{2}\right)-\frac{g_{1}}{M_{1}} \overrightarrow{\mathrm{V}}_{2} \cdot\left(\overrightarrow{\mathrm{Z}} \times \overrightarrow{\mathrm{S}}_{1}\right)\right] \\
& +\frac{g_{1} g_{2}}{4 c^{2}} \frac{Q_{1} Q_{2}}{M_{1} M_{2}} \frac{1}{Z^{3}}\left[3 \frac{\left(\overrightarrow{\mathrm{Z}} \cdot \overrightarrow{\mathrm{S}}_{1}\right)\left(\overrightarrow{\mathrm{Z}} \cdot \overrightarrow{\mathrm{S}}_{2}\right)}{Z^{2}}-\overrightarrow{\mathrm{S}}_{1} \cdot \overrightarrow{\mathrm{S}}_{2}\right] .
\end{aligned}
$$

Performing now a Legendre transformation and taking $g_{1}=g_{2}=2$ we find

$$
\begin{aligned}
H= & H_{\mathrm{DW}}+\frac{1}{2 c^{2}} \frac{Q_{1} Q_{2}}{M_{1}^{2} Z^{3}} \overrightarrow{\mathrm{P}}_{1} \cdot\left(\overrightarrow{\mathrm{Z}} \times \overrightarrow{\mathrm{S}}_{1}\right)-\frac{1}{2 c^{2}} \frac{Q_{1} Q_{2}}{M_{2}^{2} Z^{3}} \overrightarrow{\mathrm{P}}_{2} \cdot\left(\overrightarrow{\mathrm{Z}} \times \overrightarrow{\mathrm{S}}_{2}\right) \\
& +\frac{1}{c^{2}} \frac{Q_{1} Q_{2}}{M_{1} M_{2} Z^{3}}\left[\overrightarrow{\mathrm{P}}_{1} \cdot\left(\overrightarrow{\mathrm{Z}} \times \overrightarrow{\mathrm{S}}_{2}\right)-\overrightarrow{\mathrm{P}}_{2} \cdot\left(\overrightarrow{\mathrm{Z}} \times \overrightarrow{\mathrm{S}}_{1}\right)\right] \\
& +\frac{1}{c^{2}} \frac{Q_{1} Q_{2}}{M_{1} M_{2} Z^{3}}\left[\overrightarrow{\mathrm{S}}_{1} \cdot \overrightarrow{\mathrm{S}}_{2}-3 \frac{\left(\overrightarrow{\mathrm{Z}} \cdot \overrightarrow{\mathrm{S}}_{1}\right)\left(\overrightarrow{\mathrm{Z}} \cdot \overrightarrow{\mathrm{S}}_{2}\right)}{Z^{2}}\right]
\end{aligned}
$$

where $H_{\mathrm{DW}}$ stands for the Darwin Hamiltonian and $P_{1}$ and $P_{2}$ are the canonical momentums derived from (6.11) by the usual procedure. This expression coincides up to purely quantum terms and external ones with those given by other authors using the Bakamjian and Thomas formalism..$^{8-11}$ The expression (6.12) is the classical version of the Breit Hamiltonian derived in QED.

Let us now perform a similar analysis for the gravitational interaction. Multiplying (5.22) by $M_{1}$ we can write it in the form

$$
\begin{aligned}
& \frac{d}{d t}\left\{\overrightarrow{\mathrm{P}}_{1 \mathrm{EIH}}\right\} \\
&=\overrightarrow{\mathrm{F}}_{1 \mathrm{EIH}}+\frac{G}{c^{2}}\left\{-\vec{\nabla}\left[\frac{\partial}{\partial R^{r}} \frac{\partial}{\partial R^{s}}\left(\frac{1}{R}\right) S_{1}^{r} S_{2}^{s}\right]\right. \\
&-\frac{d}{d t}\left[M_{2} \frac{\overrightarrow{\mathrm{R}} \times \overrightarrow{\mathrm{S}}_{1}}{R^{3}}\right]-2 \vec{\nabla}\left[M_{2} \frac{\overrightarrow{\mathrm{R}} \cdot\left(\overrightarrow{\mathrm{V}} \times \overrightarrow{\mathrm{S}}_{1}\right)}{R^{3}}\right] \\
&\left.-2 \frac{d}{d t}\left[M_{1} \frac{\overrightarrow{\mathrm{R}} \times \overrightarrow{\mathrm{S}}_{2}}{R^{3}}\right]-2 \vec{\nabla}\left[M_{1} \frac{\overrightarrow{\mathrm{R}} \cdot\left(\overrightarrow{\mathrm{V}} \times \overrightarrow{\mathrm{S}}_{2}\right)}{R^{3}}\right]\right\},
\end{aligned}
$$

where

$$
\overrightarrow{\mathrm{P}}_{1 E I H}=\frac{\partial \mathcal{L}_{1 E I H}}{\partial \overrightarrow{\mathrm{V}}_{1}}, \overrightarrow{\mathrm{F}}_{1 E I H}=\frac{\partial \mathcal{L}_{1 E I H}}{\partial \overrightarrow{\mathrm{X}}_{1}},
$$

and $\mathscr{L}_{1 \mathrm{EIH}}$ is the Einstein-Infeld-Hoffmann Lagrangian.

Let us try again the change of variables

$$
\overrightarrow{\mathrm{Z}}_{i}=\overrightarrow{\mathrm{X}}_{i}+\frac{1}{2 c^{2}} \frac{\overrightarrow{\mathrm{V}}_{i} \times \overrightarrow{\mathrm{S}}_{i}}{M_{i}}
$$

The changes introduced can be found as before, and we have

$$
\begin{aligned}
& \frac{d}{d t}\left[\overrightarrow{\mathrm{P}}_{1 \mathrm{EIH}}+\frac{G}{c^{2}}\left(\frac{3}{2} M_{2} \frac{\overrightarrow{\mathrm{Z}} \times \overrightarrow{\mathrm{S}}_{1}}{Z^{3}}+2 M_{1} \frac{\overrightarrow{\mathrm{Z}} \times \overrightarrow{\mathrm{S}}_{2}}{Z^{3}}\right)\right]= \overrightarrow{\mathrm{F}}_{1 \mathrm{EIH}}+\frac{G}{c^{2}}\left\{-2 M_{2} \vec{\nabla}\left[\frac{\overrightarrow{\mathrm{Z}} \cdot\left(\overrightarrow{\mathrm{V}} \times \overrightarrow{\mathrm{S}}_{1}\right)}{Z^{3}}\right]-2 M_{1} \vec{\nabla}\left[\frac{\overrightarrow{\mathrm{Z}} \cdot\left(\overrightarrow{\mathrm{V}} \times \overrightarrow{\mathrm{S}}_{2}\right)}{Z^{3}}\right]\right. \\
&\left.\quad-\frac{1}{2} M_{2} \vec{\nabla}\left[\frac{\overrightarrow{\mathrm{V}}_{1} \cdot\left(\overrightarrow{\mathrm{Z}} \times \overrightarrow{\mathrm{S}}_{1}\right)}{Z^{3}}\right]+\frac{1}{2} M_{1} \vec{\nabla}\left[\frac{\overrightarrow{\mathrm{V}}_{2} \cdot\left(\overrightarrow{\mathrm{Z}} \times \overrightarrow{\mathrm{S}}_{2}\right)}{Z^{3}}\right]\right\} \\
&-\frac{G}{c^{2}}\left[\frac{\partial}{\partial Z^{r}} \frac{\partial}{\partial Z^{s}}\left(\frac{1}{Z}\right) S_{1}^{r} S_{2}^{s}\right] .
\end{aligned}
$$

As can easily be seen, this equation can be derived from the Lagrangian

$$
\mathscr{L}=\mathscr{L}_{\mathrm{EIH}}+\frac{G}{c^{2}} \frac{1}{Z^{3}}\left\{\overrightarrow{\mathrm{V}}_{1} \cdot\left[\overrightarrow{\mathrm{Z}} \times\left(\frac{3 M_{2}}{2} \overrightarrow{\mathrm{S}}_{1}+2 M_{1} \overrightarrow{\mathrm{S}}_{2}\right)\right]-\overrightarrow{\mathrm{V}}_{2} \cdot\left[\overrightarrow{\mathrm{Z}} \times\left(\frac{3 M_{1}}{2} \overrightarrow{\mathrm{S}}_{2}+2 M_{2} \overrightarrow{\mathrm{S}}_{1}\right)\right]\right\}-\frac{G}{c^{2}}\left[\frac{\partial}{\partial Z^{r}} \frac{\partial}{\partial Z^{s}}\left(\frac{1}{Z}\right) S_{1}^{r} S_{2}^{s}\right] .
$$


This Lagrangian coincides with the one derived by Barker and O'Connell ${ }^{5}$ and the equations of motion coincide, of course, at the corresponding order.

\section{CONCLUSIONS}

The results of the last section make necessary a reflection about the meaning of the coordinates $\vec{X}$ and $\vec{Q}$ we have been using.

We have on one hand a variable $\vec{X}$ possessing a set of transformation properties under the action of the Poincare group that have the same properties that points of the space have. It is also a known result that this is the unique variable we can define possessing these transformation properties. Therefore we must identify this variable wh the physical center of mass, and Eqs. (4.17)-(4.19) or $(5.20)-(5.22)$ are the ones to be used for comparison with the experimental results, in spite of the fact that they do not admit a Lagrangian form.

The variable $\vec{Q}$, as is evident from its transformation properties, cannot be identified with a position vector and therefore we cannot use it to represent the motion of a point in the space in a covariant way. This variable, however, is useful as a canonical coordinate, because as we have seen with its use the equations of motion can be put in Lagrangian form.

This reminds us strongly of a similar situation that arises in the theory of structureless interacting particles. It is a well-known result deduced from the no-interaction theorem ${ }^{12}$ that the positions cannot be used as canonical variables, that is, that the interaction among structureless particles cannot be put in Lagrangian form using the positions as canonical variables. The positions can only be used as Lagrangian variables in expansions in $v / c$ to lower orders. ${ }^{13}$

The situation we are faced with leads us to form- ulate the conjecture that in the case of spinning particles and at least for long-range interactions, $\vec{X}$ cannot be used as a canonical variable in any case. In a forthcoming paper we shall study the case of the short-range scalar interactions.

The additional term in the equation of spin in the case of the gravitational interaction can be due to the use of a flat-space metric, that therefore does not take care of the local changes of the basis vectors. This point of view is reinforced by the fact that this term does not appear in calculations performed within the framework of general relativity.

The formalism we have developed makes it possible to calculate cross sections for these interactions. This subject will be treated in the third paper of this work.

As is well known, the Einstein-Infeld-Hoffmann Lagrangian contains $G^{2}$ terms at order $1 / c^{2}$. If one uses the complete Lagrangian these terms must be treated with special care. However, under reasonable assumptions which are equivalent to the use of the Plebánski "good" $\delta$ functions, the results of Barker and O'Connell are recovered. No other special difficulties are encountered in this case, because the relations giving $\mathfrak{r}_{a}$ and $\dot{\boldsymbol{r}}_{a}$ in terms of $\overrightarrow{\mathrm{r}}_{a}$ and $\overrightarrow{\mathrm{w}}_{a}$ remain unchanged if only $c^{2}$ terms are retained.

\section{ACKNOWLEDGMENTS}

We wish to express our gratitude to Dr. J. Llosa, who has obtained the same results, except for the term in the equation of spin, from a completely different point of view, using the Einstein-Maxwell equations and finite multipolar structure, ${ }^{14}$ for allowing comparison of this work with his own, and for the active part he took in the discussions. This work was supported in part by the Instituto de Estudios Nucleares.
${ }^{1} \mathrm{X}$. Fustero and E. Verdaguer, preceding paper, Phys. Rev. D 24, 3094 (1981).

${ }^{2}$ C. F. Cho and N. D. Hari Dass, Ann. Phys. (N.Y.) 96, 406 (1976).

${ }^{3}$ B. M. Barker and R. F. O'Connell, Phys. Rev. D $\underline{12}$, 329 (1975).

${ }^{4}$ F. A. E. Pirani, Acta Phys. Pol. 15, 389 (1975).

${ }^{5}$ B. M. Barker and R. F. O'Connell, Phys. Rev. D 2 , 1428 (1970).

${ }^{6}$ V. Bargmann, L. Michel, and V. L. Telegdi, Phys . Rev. Lett. 2 , 435 (1959).

${ }^{7}$ E. Corinaldesi and A. Papapetrou, Proc. R. Soc. London
A209, 259 (1951).

${ }^{8}$ F. E. Close and H. Osborn, Phys. Rev. D 2, 2127 (1970). ${ }^{9}$ R. A. Krajcik and L. L. Foldy, Phys. Rev. D 10,1777 (1974).

${ }^{10}$ L. L. Foldy and R. A. Krajcik, Phys. Rev. D $\underline{12}, 1700$ (1975).

${ }^{11}$ F. Coester and P. Havas, Phys. Rev. D 14, 2556 (1976).

${ }^{12}$ D. G. Currie, T. F. Jordan, and E. C. G. Sudarshan, Rev. Mod. Phys. 35, 350 (1963).

${ }^{13}$ J. Martín and J. L. Sanz, J. Math. Phys. 19, 4 (1978).

${ }^{14} \mathrm{~J}$. Llosa, Ph.D. thesis, University of Barcelona, 1978 (unpublished). 